ESAIM: COCV 18 (2012) 930-940

DOI: $10.1051 / \mathrm{cocv} / 2011189$
ESAIM: Control, Optimisation and Calculus of Variations

www.esaim-cocv.org

\title{
MULTIPLICITY OF SOLUTIONS FOR THE NONCOOPERATIVE $p$-LAPLACIAN OPERATOR ELLIPTIC SYSTEM WITH NONLINEAR BOUNDARY CONDITIONS
}

\author{
SinuA Liang ${ }^{1}$ AND JiHui Zhang ${ }^{2}$
}

\begin{abstract}
In this paper, we study the multiplicity of solutions for a class of noncooperative $p$-Laplacian operator elliptic system. Under suitable assumptions, we obtain a sequence of solutions by using the limit index theory.
\end{abstract}

Mathematics Subject Classification. 35J70, 35B20.

Received May 5, 2011.

Published online 16 January 2012.

\section{INTRODUCTION}

In this paper we deal with the existence and multiplicity of solutions to the following $p$-Laplacian operator elliptic system with nonlinear boundary conditions.

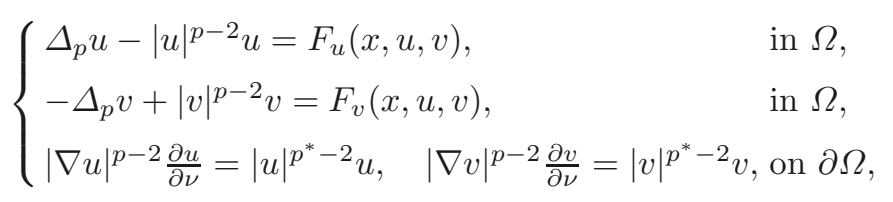

where $1<p<N, \Omega \subset \mathbb{R}^{N}(N \geq 3)$ is a bounded domain with smooth boundary, $\Delta_{p} u:=\operatorname{div}\left(|\nabla u|^{p-2} \nabla u\right)$ is a $p$-Laplacian operator and $\frac{\partial}{\partial \nu}$ is the outer normal derivative, $F=F(x, u, v), F_{u}=\frac{\partial F}{\partial u}, F_{v}=\frac{\partial F}{\partial v}, p^{*}=N p /(N-p)$ is the critical exponent according to the Sobolev embedding.

In recent years, the existence and multiplicity of solutions for a noncooperative elliptic system have been obtained by many papers. In [1], Benci assumed $X$ is a Hilbert space, $f$ satisfies $(P S)$-condition and is the form

$$
f(u)=\frac{1}{2}\langle L u, u\rangle+\Phi(u),
$$

where $L$ is bounded self-adjoint operator and $\Phi^{\prime}$ is compact.

Keywords and phrases. $p$-Laplacian operator, limit index, critical growth, concentration-compactness principle.

1 College of Mathematics, Changchun Normal University, Changchun 130032, Jilin, P.R. China. liangsihua@163.com

2 Jiangsu Key Laboratory for NSLSCS, School of Mathematical Sciences, Nanjing Normal University, Nanjing, Jiangsu 210046,

P.R. China. jihuiz@jlonline.com 
When $p=2$ (a constant) with Dirichlet boundary condition, Lin and Li [9] considered the following system

$$
\begin{cases}\Delta u=|u|^{2^{*}-2} u+F_{u}(x, u, v), & \text { in } \Omega, \\ -\Delta v=|v|^{2^{*}-2} v+F_{v}(x, u, v), & \text { in } \Omega, \\ u=0, \quad v=0, & \text { on } \partial \Omega,\end{cases}
$$

by applying the Limit Index Theory, they obtained the existence of multiple solutions under some assumptions on nonlinear part.

When $p \neq 2$, Huang and Li [6] considered the following the system of elliptic equations involving the $p$-Laplacian in the unbounded domain of $\mathbb{R}^{N}$ by applying the Limit Index Theory,

$$
\begin{cases}\Delta_{p} u-|u|^{p-2} u=F_{u}(x, u, v), & \text { in } \mathbb{R}^{N}, \\ -\Delta_{p} v+|v|^{p-2} v=F_{v}(x, u, v), & \text { in } \mathbb{R}^{N}, \\ u, v \in W^{1, p}\left(\mathbb{R}^{N}\right), & \end{cases}
$$

where $1<p<N$ and they extended some results of [8].

We note that these papers deal with Dirichlet boundary condition $[2,7]$. However, nonlinear boundary conditions have only been considered in recent years. For the Laplace operator with nonlinear boundary conditions see for example [3,14]. For elliptic systems with nonlinear boundary conditions see [5]. For previous work for the $p$-Laplacian with nonlinear boundary conditions of different type see $[4,13]$.

Motivated by papers above, a natural question arises whether the existence and multiplicity of solutions to the $p$-Laplacian operator elliptic system with nonlinear boundary conditions (1.1) can be obtained. In this paper we deal with the problem (1.1). Throughout this paper, we assume that $F(x, u, v)$ satisfies the following conditions:

$\left(\mathrm{H}_{1}\right) F \in C\left(\bar{\Omega} \times R^{2}, R^{+}\right)$and $F(x, s, t)=F(x,-s,-t)$ for all $(x, s, t) \in \Omega \times \in \mathbb{R}^{2}$;

$\left(\mathrm{H}_{2}\right) \lim _{|t| \rightarrow \infty} \frac{F_{t}(x, s, t)}{|t|^{p-1}}=0$ uniformly for $x \in \Omega$;

$\left(\mathrm{H}_{3}\right) s F_{s}(x, s, t) \geq 0$ for all $(x, s, t) \in \bar{\Omega} \times R^{2}$.

Under assumptions $\left(\mathrm{H}_{1}\right)$ and $\left(\mathrm{H}_{2}\right)$, we have

$$
F_{v}(x, u, v) v=o\left(|v|^{p}\right),
$$

which means that, for all $\varepsilon>0$, there exist $a(\varepsilon), b(\varepsilon)>0$ such that

$$
|F(x, 0, v) v| \leq a(\varepsilon)+\varepsilon|v|^{p}
$$

and

$$
\left|F_{v}(x, u, v) v\right| \leq b(\varepsilon)+\varepsilon|v|^{p} .
$$

Hence, together with condition (1.2), (1.3) and the mean value theorem for any constants $\beta$ and fixed $u$ we have

$$
\left|F(x, u, v)-\beta F_{v}(x, u, v) v\right| \leq c(\varepsilon)+\varepsilon|v|^{p},
$$

for some $c(\varepsilon)>0$.

Furthermore, we assume that $F(x, u, v)$ satisfies condition:

$\left(\mathrm{H}_{4}\right)$ There exist $L>0$ (where $L$ will be determined later) and

$$
\xi<|\Omega|^{-1} \min \left\{0, \frac{1}{N} S^{p^{*} /\left(p^{*}-p\right)}-c\left(\frac{1}{2 N}\right)|\Omega|\right\}
$$

such that $F(x, s, t) t \geq L|t|^{p}-\xi$, for every $(x, s, t) \in \bar{\Omega} \times \mathbb{R}^{2}$. 
Notation. Weak (resp. strong) convergence is denoted by $\rightarrow$ (resp., $\rightarrow$ ). $|\cdot|_{p}$ is the usual norm in $L^{p}(\Omega)$. $L_{2}^{p}(\Omega)=L^{p}(\Omega) \times L^{p}(\Omega)$ with the norm $|(u, v)|_{p}:=\left(|u|_{p}^{p}+|v|_{p}^{p}\right)^{\frac{1}{p}} . E:=W^{1, p}(\Omega)$ with the norm $\|u\|_{p}:=$ $\int_{\Omega}\left(|\nabla u|^{p}+|u|^{p}\right) \mathrm{d} x . Y=E \times E, X_{n}=E \times E_{n} . c_{i}$ denote a positive constant and can be determined in concrete conditions.

According to [15], there exists a Schauder basis $\left\{e_{n}\right\}_{n=1}^{\infty}$ for $E$. Furthermore, since $E$ is reflexive, $\left\{e_{n}^{*}\right\}_{n=1}^{\infty}$ the biorthogonal functionals associated to the basis $\left\{e_{n}\right\}_{n=1}^{\infty}$ which are characterized by the relations

$$
e_{n}^{*}\left(e_{m}\right)=\delta_{n, m}= \begin{cases}1, & \text { if } n=m \\ 0, & \text { if } n \neq m\end{cases}
$$

form a basis for $E^{*}$ with the following properties (cf. [10] Prop. 1.b.1 and Thm. 1.b.5). Denote

$$
E_{n}=\operatorname{span}\left\{e_{1}, \ldots, e_{n}\right\}, \quad E_{n}^{\perp}=\overline{\operatorname{span}\left\{e_{n+1}, \ldots\right\}}
$$

and

$$
E_{n}^{*}=\operatorname{span}\left\{e_{1}^{*}, \ldots, e_{n}^{*}\right\} .
$$

Let $P_{n}: E \rightarrow E_{n}$ be the projector corresponding to decomposition $E=E_{n} \oplus E_{n}^{\perp}$ and $P_{n}^{*}: E^{*} \rightarrow E_{n}^{*}$ be the projector corresponding to the decomposition $E^{*}=E_{n}^{*} \oplus\left(E_{n}^{*}\right)^{\perp}$. Then $P_{n} u \rightarrow u, P_{n}^{*} v^{*} \rightarrow v^{*}$ for any $u \in E$, $v^{*} \in E^{*}$ as $n \rightarrow \infty$ and $\left\langle P_{n}^{*} v^{*}, u\right\rangle=\left\langle v^{*}, P_{n} u\right\rangle$. Let $\tau: E \rightarrow E^{*}$ be the mapping given by

$$
\langle\tau u, \widetilde{u}\rangle=\int_{\Omega}|\nabla u|^{p-2} \nabla u \cdot \nabla \widetilde{u} \mathrm{~d} x, \quad \text { for all } u, \widetilde{u} \in E .
$$

It is easy to check that the operator $\tau$ is bounded, continuous. And if $u_{n} \rightarrow \widetilde{u}$ in $E$ and $\left\langle\tau u_{n}-\tau \widetilde{u}, u_{n}-\widetilde{u}\right\rangle \rightarrow 0$, then $u_{n} \rightarrow \widetilde{u}$ in $E($ see $[6,8])$

The energy functional corresponding to problem (1.1) is defined as follows,

$$
\begin{aligned}
J(u, v)= & -\frac{1}{p} \int_{\Omega}\left(|\nabla u|^{p}+|u|^{p}\right) \mathrm{d} x+\frac{1}{p} \int_{\Omega}\left(|\nabla v|^{p}+|v|^{p}\right) \mathrm{d} x \\
& -\frac{1}{p^{*}} \int_{\partial \Omega}|u|^{p^{*}} \mathrm{~d} \sigma-\frac{1}{p^{*}} \int_{\partial \Omega}|v|^{p^{*}} \mathrm{~d} \sigma-\int_{\Omega} F(x, u, v) \mathrm{d} x .
\end{aligned}
$$

The main result of this paper is as follows.

Theorem 1.1. Suppose that $F(x, u, v)$ satisfies conditions $\left(H_{1}\right)-\left(H_{4}\right)$. Then there exists $k_{0}>1$ such that (1.1) possesses at least $k_{0}-1$ pairs weak nontrivial solutions.

Remark 1.2. There are two difficulties in considering the elliptic problem (1.1). One is the functional $J(u, v)$ is strongly indefinite. Therefore one cannot apply the symmetric Mountain pass theorem of the functional $J(u, v)$. The other one in solving the problem is a lack of compactness which can be illustrated by the fact that the embedding of $W^{1, p}(\Omega)$ into $L^{p^{*}}(\partial \Omega)$ is no longer compact.

Remark 1.3. Theorem 1.1 is new as far as we know. We mainly follow the way in [8] to prove our main result.

\section{PRELiminaRies AND LEMmas}

First of all, we recall the limit index theory due to $\mathrm{Li}$ [8]. In order to do that, we introduce the following definitions.

Definition 2.1. $[8,16]$ The action of a topological group $G$ on a normed space $Z$ is a continuous map

$$
G \times Z \rightarrow Z:[g, z] \mapsto g z
$$


such that

$$
1 \cdot z=z, \quad(g h) z=g(h z) \quad z \mapsto g z \text { is linear, } \forall g, h \in G .
$$

The action is isometric if

$$
\|g z\|=\|z\|, \quad \forall g \in G, \quad z \in Z .
$$

And in this case $Z$ is called $G$-space.

The set of invariant points is defined by

$$
\operatorname{Fix} G:=\{z \in Z: g z=z, \forall g \in G\} .
$$

A set $A \subset Z$ is invariant if $g A=A$ for every $g \in G$. A function $\varphi: Z \rightarrow R$ is invariant $\varphi \circ g=\varphi$ for every $g \in G, z \in Z$. A map $f: Z \rightarrow Z$ is equivariant if $g \circ f=f \circ g$ for every $g \in G$.

Suppose $Z$ is a $G$-Banach space, that is, there is a $G$ isometric action on $Z$. Let

$$
\Sigma:=\{A \subset Z: A \text { is closed and } g A=A, \forall g \in G\}
$$

be a family of all $G$-invariant closed subset of $Z$, and let

$$
\Gamma:=\left\{h \in C^{0}(Z, Z): h(g u)=g(h u), \quad \forall g \in G\right\}
$$

be the class of all $G$-equivariant mapping of $Z$. Finally, we call the set

$$
O(u):=\{g u: g \in G\}
$$

$G$-orbit of $u$.

Definition 2.2. [8] An index for $(G, \Sigma, \Gamma)$ is a mapping $i: \Sigma \rightarrow \mathcal{Z}_{+} \cup\{+\infty\}$ (where $\mathcal{Z}_{+}$is the set of all nonnegative integers) such that for all $A, B \in \Sigma, h \in \Gamma$, the following conditions are satisfied:

(1) $i(A)=0 \Leftrightarrow A=\emptyset$;

(2) (monotonicity) $A \subset B \Rightarrow i(A) \leq i(B)$;

(3) (subadditivity) $i(A \cup B) \leq i(A)+i(B)$;

(4) (supervariance) $i(A) \leq i(\overline{h(A)}), \forall h \in \Gamma$;

(5) (continuity) If $A$ is compact and $A \cap \operatorname{Fix} G=\emptyset$, then $i(A)<+\infty$ and there is a $G$-invariant neighbourhood $N$ of $A$ such that $i(\bar{N})=i(A)$;

(6) (normalization) If $x \notin \operatorname{Fix} G$, then $i(O(x))=1$.

Definition 2.3. [1] An index theory is said to satisfy the $d$-dimension property if there is a positive integer $d$ such that

$$
i\left(V^{d k} \cap S_{1}\right)=k
$$

for all $d k$-dimensional subspaces $V^{d k} \in \Sigma$ such that $V^{d k} \cap \operatorname{Fix} G=\{0\}$, where $S_{1}$ is the unit sphere in $Z$.

Suppose $U$ and $V$ are $G$-invariant closed subspaces of $Z$ such that

$$
Z=U \oplus V,
$$

where $V$ is infinite dimensional and

$$
V=\overline{\bigcup_{j=1}^{\infty} V_{j}}
$$


where $V_{j}$ is a $d n_{j}$-dimensional $G$-invariant subspace of $V, j=1,2, \ldots$, and $V_{1} \subset V_{2} \subset \ldots \subset V_{n} \subset \ldots$ Let

$$
Z_{j}=U \bigoplus V_{j}
$$

and $\forall A \in \Sigma$, let

$$
A_{j}=A \bigoplus Z_{j}
$$

Definition 2.4. [8] Let $i$ be an index theory satisfying the $d$-dimension property. A limit index with respect to $\left(Z_{j}\right)$ induced by $i$ is a mapping

$$
i^{\infty}: \Sigma \rightarrow \mathcal{Z} \cup\{-\infty,+\infty\}
$$

given by

$$
i^{\infty}(A)=\lim \sup _{j \rightarrow \infty}\left(i\left(A_{j}\right)-n_{j}\right) .
$$

Proposition 2.5. [8] Let $A, B \in \Sigma$. Then $i^{\infty}$ satisfies:

(1) $A=\emptyset \Rightarrow i^{\infty}=-\infty$;

(2) (monotonicity) $A \subset B \Rightarrow i^{\infty}(A) \leq i^{\infty}(B)$;

(3) (subadditivity) $i^{\infty}(A \cup B) \leq i^{\infty}(A)+i^{\infty}(B)$;

(4) If $V \cap \operatorname{Fix} G=\{0\}$, then $i^{\infty}\left(S_{\rho} \cap V\right)=0$, where $S_{\rho}=\{z \in Z:\|z\|=\rho\}$;

(5) If $Y_{0}$ and $\widetilde{Y_{0}}$ are $G$-invariant closed subspaces of $V$ such that $V=Y_{0} \oplus \widetilde{Y_{0}}, \widetilde{Y_{0}} \subset V_{j_{0}}$ for some $j_{0}$ and $\operatorname{dim} \widetilde{Y_{0}}=d m$, then $i^{\infty}\left(S_{\rho} \cap Y_{0}\right) \geq-m$.

Definition 2.6. [16] A functional $J \in C^{1}(Z, R)$ is said to satisfy the condition $(P S)_{c}^{*}$ if any sequence $\left\{u_{n_{k}}\right\}$, $u_{n_{k}} \in Z_{n_{k}}$ such that

$$
J\left(u_{n_{k}}\right) \rightarrow c, \quad \mathrm{~d} J_{n_{k}}\left(u_{n k}\right) \rightarrow 0, \quad \text { as } k \rightarrow \infty
$$

possesses a convergent subsequence, where $Z_{n_{k}}$ is the $n_{k}$-dimension subspace of $Z, J_{n_{k}}=\left.J\right|_{Z_{n_{k}}}$.

Theorem 2.7. [8] Assume that

$\left(\mathrm{B}_{1}\right) J \in C^{1}(Z, R)$ is $G$-invariant;

$\left(\mathrm{B}_{2}\right)$ there are $G$-invariant closed subspaces $U$ and $V$ such that $V$ is infinite dimensional and $Z=U \oplus V$;

$\left(\mathrm{B}_{3}\right)$ there is a sequence of $G$-invariant finite dimensional subspaces

$$
V_{1} \subset V_{2} \subset \cdots \subset V_{j} \subset \cdots, \quad \operatorname{dim} V_{j}=d n_{j},
$$

such that $V=\overline{\cup_{j=1}^{\infty} V_{j}}$;

$\left(\mathrm{B}_{4}\right)$ there is an index theory $i$ on $Z$ satisfying the d-dimension property;

$\left(\mathrm{B}_{5}\right)$ there are $G$-invariant subspaces $Y_{0}, \widetilde{Y_{0}}, Y_{1}$ of $V$ such that $V=Y_{0} \oplus \widetilde{Y_{0}}, Y_{1}, \widetilde{Y_{0}} \subset V_{j_{0}}$ for some $j_{0}$ and $\operatorname{dim} \widetilde{Y_{0}}=\mathrm{d} m<\mathrm{d} k=\operatorname{dim} Y_{1} ;$

$\left(\mathrm{B}_{6}\right)$ there are $\alpha$ and $\beta, \alpha<\beta$ such that $f$ satisfies $(P S)_{c}^{*}, \forall c \in[\alpha, \beta]$;

$\left(\mathrm{B}_{7}\right)$

$$
\left\{\begin{array}{l}
\text { (a) either Fix } G \subset U \oplus Y_{1}, \quad \text { or } \quad \text { Fix } G \cap V=\{0\}, \\
\text { (b) there is } \rho>0 \text { such that } \forall u \in Y_{0} \cap S_{\rho}, f(z) \geq \alpha, \\
\text { (c) } \forall z \in U \oplus Y_{1}, f(z) \leq \beta,
\end{array}\right.
$$

if $i^{\infty}$ is the limit index corresponding to $i$, then the numbers

$$
c_{j}=\inf _{i \infty} \sup _{(A) \geq j} f(u), \quad-k+1 \leq j \leq-m,
$$

are critical values of $f$, and $\alpha \leq c_{-k+1} \leq \cdots \leq c_{-m} \leq \beta$. Moreover, if $c=c_{l}=\cdots=c_{l+r}, r \geq 0$, then $i\left(\mathbb{K}_{c}\right) \geq r+1$, where $\mathbb{K}_{c}=\{z \in Z: \mathrm{d} f(z)=0, f(z)=c\}$. 


\section{Local Palais-Smale Condition}

To prove Theorem 1.1, noting the lack of compactness, in the inclusion $W^{1, p}(\Omega) \hookrightarrow L^{p^{*}}(\partial \Omega)$, we can no longer expect the Palais-Smale condition to hold. Anyway we can prove a local Palais-Smale condition that will hold for $J(u, v)$ below a certain value of energy. Let $u_{n}$ be a bounded sequence in $W^{1, p}(\Omega)$ then there exists a subsequence that we still denote $u_{n}$ such that

$$
\begin{aligned}
& u_{n} \rightarrow u \quad \text { weakly in } W^{1, p}(\Omega), \\
& u_{n} \rightarrow u \quad \text { strongly in } L^{r}(\Omega), 1 \leq r<p^{*}, \\
& \left|\nabla u_{n}\right|^{p} \rightarrow \mathrm{d} \mu,\left.\quad\left|u_{n}\right|_{\partial \Omega}\right|^{p^{*}} \rightarrow \mathrm{d} \eta,
\end{aligned}
$$

weakly-* $^{*}$ in the sense of measures. Observe that $\mathrm{d} \eta$ is a measure supported on $\partial \Omega$.

If we consider $\phi \in C^{\infty}(\bar{\Omega})$, from the Sobolev trace inequality we obtain, passing to the limit

$$
\left(\int_{\partial \Omega}|\phi|^{p^{*}} \mathrm{~d} \eta\right)^{\frac{1}{p^{*}}} S^{\frac{1}{p}} \leq\left(\int_{\Omega}|\phi|^{p} \mathrm{~d} \mu+\int_{\Omega}|u|^{p}|\nabla \phi|^{p} \mathrm{~d} x+\int_{\Omega}|\phi u|^{p} \mathrm{~d} x\right)^{\frac{1}{p}},
$$

where $S$ is the best constant in the Sobolev trace embedding theorem. From (3.1) we observe that if $u=0$ we get a reverse Hölder-type inequality (but it involves one integral over $\Omega$ ) between the two measures $\mu$ and $\eta$.

Similar to the proof of $[11,12]$, we have the following lemma.

Lemma 3.1. [4] Let $u_{j}$ be a weakly convergent sequence in $W^{1, p}(\Omega)$ with weak limit $u$ such that

$$
\left|\nabla u_{j}\right|^{p} \rightarrow \mathrm{d} \mu, \quad \text { and }\left.\quad\left|u_{j}\right|_{\partial \Omega}\right|^{p^{*}} \rightarrow \mathrm{d} \eta,
$$

weakly-* in the sense of measures. Then there exists $x_{1}, \ldots, x_{l} \in \partial \Omega$ such that

(i) $\mathrm{d} \eta=|u|^{p^{*}}+\sum_{j=1}^{l} \eta_{j} \delta_{x_{j}}, \eta_{j}>0$;

(ii) $\mathrm{d} \mu \geq|\nabla u|^{p}+\sum_{j=1}^{l} \mu_{j} \delta_{x_{j}}, \mu_{j}>0$;

(iii) $\left(\eta_{j}\right)^{\frac{p}{p^{*}}} \leq \frac{\mu_{j}}{S}$.

Similar to $[6,16]$, it is easy to obtain the following lemma:

Lemma 3.2. Assume $1 \leq \theta_{1}, \theta_{2}, \theta<\infty, I \in C\left(\bar{\Omega} \times R^{2}, R\right)$ and

$$
I(x, u, v) \leq C\left(|u|^{\frac{\theta_{1}}{\theta}}+|v|^{\frac{\theta_{2}}{\theta}}\right) .
$$

Then for every $(u, v) \in L^{\theta_{1}}(\Omega) \times L^{\theta_{2}}(\Omega), I(\cdot, u, v) \in L^{\theta}(\Omega)$ and the operator

$$
T:(u, v) \mapsto I(x, u, v)
$$

is a continuous map from $L^{\theta_{1}}(\Omega) \times L^{\theta_{2}}(\Omega)$ to $L^{\theta}(\Omega)$.

Lemma 3.3. Suppose that $F(x, u, v)$ satisfies conditions $\left(H_{1}\right)-\left(H_{3}\right)$. Then

(i) $J \in C^{1}(X, R)$;

(ii)

$$
\begin{aligned}
\langle\mathrm{d} J(u, v),(\widehat{u}, \widehat{v})\rangle= & -\int_{\Omega}|\nabla u|^{p-2} \nabla u \cdot \nabla \widehat{u}+|u|^{p-2} u \widehat{u} \mathrm{~d} x-\int_{\partial \Omega}|u|^{p^{*}-2} u \widehat{u} \mathrm{~d} \sigma \\
& +\int_{\Omega}|\nabla v|^{p-2} \nabla v \cdot \nabla \widehat{v}+\ll|v|^{p-2} v \widehat{v} \mathrm{~d} x-\int_{\partial \Omega}|v|^{p^{*}-2} v \widehat{v} \mathrm{~d} \sigma \\
& -\int_{\Omega} F_{u}(x, u, v) \widehat{u} \mathrm{~d} x-\int_{\Omega} F_{v}(x, u, v) \widehat{v} \mathrm{~d} x ;
\end{aligned}
$$

(iii) A critical point of $J$ is a weak solution of system (1.1). 
Now set

$$
\begin{gathered}
X=U \oplus V, \quad U=E \times\{0\}, \quad V=\{0\} \times E, \\
Y_{0}=\{0\} \times E_{1}^{\perp}, \quad V=Y_{0} \oplus \widetilde{Y_{0}}, \\
Y_{1}=\{0\} \times E_{k_{0}}, \quad E_{k_{0}}=\operatorname{span}\left\{e_{1}, \ldots, e_{k_{0}}\right\},
\end{gathered}
$$

then $\operatorname{dim} \widetilde{Y_{0}}=1, \operatorname{dim} Y_{1}=k_{0}$.

Define a group action $G_{2}=\{1, \tau\} \cong \mathcal{Z}_{2}$ by setting $\tau(u, v)=(-u,-v)$, then Fix $G=\{0\} \times\{0\}$ (also denote $\{0\})$. It is clear that $U$ and $V$ are $G$-invariant closed subspaces of $X$, and $Y_{0}, \widetilde{Y_{0}}$ and $Y_{1}$ are $G$-invariant subspace of $V$. Set

$$
\Sigma:=\{A \subset X \backslash\{0\}: A \text { is closed in } X \text { and }(u, v) \in A \Rightarrow(-u,-v) \in A\} .
$$

Define an index $\gamma$ on $\Sigma$ by:

$$
\gamma(A)=\left\{\begin{array}{l}
\min \left\{N \in \mathcal{Z}: \exists h \in C\left(A, \mathbb{R}^{N} \backslash\{0\} \text { such that } h(-u,-v)=h(u, v)\right)\right\}, \\
0, \quad \text { if } A=\emptyset, \\
+\infty, \quad \text { if such } h \text { does not exist. }
\end{array}\right.
$$

Then we have the following proposition from [6]: $\gamma$ is an index satisfying the properties given in Definition 2.2. Moreover, $\gamma$ satisfies the one-dimension property. According to Definition 2.4 we can obtain a limit index $\gamma^{\infty}$ with respect to $\left(X_{n}\right)$ from $\gamma$.

Now we turn to prove local Palais-Smale condition.

Lemma 3.4. Assume condition $\left(H_{1}\right)-\left(H_{3}\right)$ hold, Then the functional $J$ satisfies the local $(P S)_{c}$ condition in

$$
c \in\left(-\infty, \frac{1}{N} S^{p^{*} /\left(p^{*}-p\right)}-c\left(\frac{1}{2 N}\right)|\Omega|\right),
$$

in the following sense: if

$$
J\left(u_{n_{k}}, v_{n_{k}}\right) \rightarrow c \in\left(-\infty, \frac{1}{N} S^{p^{*} /\left(p^{*}-p\right)}-c\left(\frac{1}{2 N}\right)|\Omega|\right), \quad \mathrm{d} J_{n_{k}}\left(u_{n_{k}}, v_{n_{k}}\right) \rightarrow 0, \quad \text { as } k \rightarrow \infty,
$$

where $J_{n_{k}}=\left.J\right|_{X_{n_{k}}}$. Then $\left\{\left(u_{n_{k}}, v_{n_{k}}\right)\right\}$ contains a subsequence converging strongly in $X$.

Proof. First, we show that $\left\{\left(u_{n_{k}}, v_{n_{k}}\right)\right\}$ is bounded in $X$.

We note that by condition $\left(H_{3}\right)$,

$$
\begin{aligned}
o(1)\left\|u_{n_{k}}\right\|_{p} & \geq\left\langle-\mathrm{d} J_{n_{k}}\left(u_{n_{k}}, v_{n_{k}}\right),\left(u_{n_{k}}, 0\right)\right\rangle \\
& =\int_{\Omega}\left|\nabla u_{n_{k}}\right|^{p}+\left|u_{n_{k}}\right|^{p} \mathrm{~d} x+\int_{\partial \Omega}\left|u_{n_{k}}\right|^{p^{*}} \mathrm{~d} \sigma+\int_{\Omega} F_{u}\left(x, u_{n_{k}}, v_{n_{k}}\right) u_{n_{k}} \mathrm{~d} x \\
& \geq \int_{\Omega}\left|\nabla u_{n_{k}}\right|^{p}+\left|u_{n_{k}}\right|^{p} \mathrm{~d} x+\int_{\partial \Omega}\left|u_{n_{k}}\right|^{p^{*}} \mathrm{~d} x \\
& \geq\left\|u_{n_{k}}\right\|_{p}^{p},
\end{aligned}
$$

since $p>1$, from (3.2), we know that $\left\|u_{n_{k}}\right\|_{p}$ is bounded. On the one hand, we have

$$
\begin{aligned}
& J_{n_{k}}\left(0, v_{n_{k}}\right)-\frac{1}{p^{*}}\left\langle\mathrm{~d} J_{n_{k}}\left(u_{n_{k}}, v_{n_{k}}\right),\left(0, v_{n_{k}}\right)\right\rangle \\
= & \left(\frac{1}{p}-\frac{1}{p^{*}}\right) \int_{\Omega}\left(\left|\nabla v_{n_{k}}\right|^{p}+\left|v_{n_{k}}\right|^{p}\right) \mathrm{d} x-\int_{\Omega}\left[F\left(x, u_{n_{k}}, v_{n_{k}}\right)-\frac{1}{p^{*}} F_{v}\left(x, u_{n_{k}}, v_{n_{k}}\right) v_{n_{k}}\right] \mathrm{d} x \\
= & c+o(1)\left\|v_{n}\right\|_{p},
\end{aligned}
$$


i.e.

$$
\begin{aligned}
\frac{1}{N} \int_{\Omega}\left(\left|\nabla v_{n_{k}}\right|^{p}+\left|v_{n_{k}}\right|^{p}\right) \mathrm{d} x= & \int_{\Omega}\left[F\left(x, u_{n_{k}}, v_{n_{k}}\right)-\frac{1}{p^{*}} F_{v}\left(x, u_{n_{k}}, v_{n_{k}}\right) v_{n_{k}}\right] \mathrm{d} x \\
& +c+o(1)\left\|v_{n_{k}}\right\|_{p} .
\end{aligned}
$$

Then by (1.4), we have

$$
\left(\frac{1}{N}-\varepsilon\right)\left\|v_{n_{k}}\right\|_{p}^{p} \leq c(\varepsilon)|\Omega|+c+o(1)\left\|v_{n_{k}}\right\|_{p}
$$

where $|\cdot|$ denote by Lebesgue measure. Setting $\varepsilon=1 / 2 N$, we get

$$
\left\|v_{n_{k}}\right\|_{p}^{p} \leq M+o(1)\left\|v_{n_{k}}\right\|_{p}
$$

where $o(1) \rightarrow 0$ and $M$ is a some positive number. Thus (3.3) implies that $\left\{v_{n_{k}}\right\}$ is bounded in $W^{1, p}(\Omega)$. This implies $\left\|u_{n_{k}}\right\|_{p}+\left\|v_{n_{k}}\right\|_{p}$ is bounded in $X$.

Next, we prove that $\left\{\left(u_{n_{k}}, v_{n_{k}}\right)\right\}$ contains a subsequence converging strongly in $X$.

We note that $\left\{u_{n_{k}}\right\}$ is bounded in $E$. Hence, up to a subsequence, $u_{n_{k}} \rightarrow u$ weakly in $E$ and $u_{n_{k}}(x) \rightarrow u(x)$, a.e. in $\mathbb{R}^{N}$. We claim that $u_{n_{k}} \rightarrow u$ strongly in $E$. In fact, note that

$$
\begin{aligned}
\int_{\Omega}\left|\nabla u_{n_{k}}-\nabla u\right|^{p}+\left|u_{n_{k}}-u\right|^{p} \mathrm{~d} x+\int_{\partial \Omega}\left|u_{n_{k}}-u\right|^{p^{*}} \mathrm{~d} \sigma & +\int_{\Omega} F_{u}\left(x, u_{n_{k}}-u, v_{n_{k}}\right)\left(u_{n_{k}}-u\right) \mathrm{d} x \\
& =\left\langle-\mathrm{d} J_{n_{k}}\left(u_{n_{k}}-u, v_{n_{k}}\right),\left(u_{n_{k}}-u, 0\right)\right\rangle \rightarrow 0 \quad \text { as } n \rightarrow \infty,
\end{aligned}
$$

and condition $\left(H_{3}\right)$ imply that

$$
u_{n_{k}} \rightarrow u \quad \text { strongly in } E \text {. }
$$

In the following we will prove that there exists $v \in E$ such that

$$
v_{n_{k}} \rightarrow v \quad \text { strongly in } E \text {. }
$$

By Lemma 3.1 and (3.3) there exists a subsequence, there exists a subsequence, that we still denote $v_{n_{k}}$ such that

$$
\begin{aligned}
& v_{n_{k}} \rightarrow v \quad \text { weakly in } W^{1, p}(\Omega), \\
& v_{n_{k}} \rightarrow v \quad \text { strongly in } L^{r}(\Omega), 1 \leq r<p^{*}, \text { and a.e. in } \Omega \\
& \left|\nabla v_{n_{k}}\right|^{p} \rightarrow \mathrm{d} \mu \geq|\nabla v|^{p}+\sum_{k=1}^{l} \mu_{k} \delta_{x_{k}} \\
& \left.\left|v_{n_{k}}\right|_{\partial \Omega}\right|^{p^{*}} \rightarrow \mathrm{d} \eta=\left.|v|_{\partial \Omega}\right|^{p^{*}}+\sum_{k=1}^{l} \eta_{k} \delta_{x_{k}} .
\end{aligned}
$$

Let $\phi(x) \in C^{\infty}(\Omega)$ such that $\phi(x) \equiv 1$ in $B\left(x_{k}, \varepsilon\right), \phi(x) \equiv 0$ in $\Omega \backslash\left(x_{k}, 2 \varepsilon\right)$ and $|\nabla \phi| \leq 2 / \varepsilon$, where $x_{k}$ belongs to the support of $\mathrm{d} \eta$. Consider Then $\left\{\phi v_{n_{k}}\right\}$ is bounded in $E$, Obviously, $\left\langle\mathrm{d} J_{n_{k}}\left(u_{n_{k}}, v_{n_{k}}\right),\left(0, v_{n_{k}} \phi\right)\right\rangle \rightarrow 0$, i.e.

$$
\begin{aligned}
& \lim _{n \rightarrow \infty}\left[\int_{\Omega}\left(\left|\nabla v_{n_{k}}\right|^{p}+\left|v_{n_{k}}\right|^{p}\right) \phi \mathrm{d} x-\int_{\partial \Omega}\left|v_{n_{k}}\right|^{p^{*}} \phi \mathrm{d} \sigma-\int_{\Omega} F_{v}\left(x, u_{n_{k}}, v_{n_{k}}\right) v_{n_{k}} \phi \mathrm{d} x\right] \\
& =-\lim _{n \rightarrow \infty} \int_{\Omega}\left(v_{n_{k}}\left|\nabla v_{n_{k}}\right|^{p-2} \nabla v_{n_{k}} \nabla \phi\right) \mathrm{d} x .
\end{aligned}
$$


On the other hand, by Hölder inequality and weak convergence, we obtain

$$
\begin{aligned}
0 & \leq\left.\lim _{\varepsilon \rightarrow 0} \lim _{n \rightarrow \infty}\left|\int_{\Omega} v_{n_{k}}\right| \nabla v_{n_{k}}\right|^{p-2} \nabla v_{n_{k}} \nabla \phi \mathrm{d} x \mid \\
& \leq \lim _{\varepsilon \rightarrow 0} \lim _{n \rightarrow \infty}\left(\int_{\Omega}\left|v_{n_{k}}\right|^{p}|\nabla \phi|^{p} \mathrm{~d} x\right)^{\frac{1}{p}}\left(\int_{\Omega}\left|\nabla v_{n_{k}}\right|^{q} \mathrm{~d} x\right)^{\frac{p-1}{p}} \\
& \leq C \lim _{\varepsilon \rightarrow 0}\left(\int_{\Omega}|v|^{p}|\nabla \phi|^{p} \mathrm{~d} x\right)^{\frac{1}{p}} \\
& \leq C \lim _{\varepsilon \rightarrow 0}\left(\int_{B\left(x_{j}, \varepsilon\right)}|\nabla \phi|^{N} \mathrm{~d} x\right)^{\frac{1}{N}}\left(\int_{B\left(x_{j}, \varepsilon\right)}|v|^{p^{*}} \mathrm{~d} x\right)^{\frac{1}{p^{*}}} \\
& \leq C \lim _{\varepsilon \rightarrow 0}\left(\int_{B\left(x_{j}, \varepsilon\right)}|v|^{p^{*}} \mathrm{~d} x\right)^{\frac{1}{p^{*}}}=0 .
\end{aligned}
$$

From (3.6) and (3.7), we have

$$
0=\lim _{\varepsilon \rightarrow 0}\left[\int_{\partial \Omega} \phi \mathrm{d} \eta-\int_{\Omega} \phi \mathrm{d} \mu-\int_{\Omega}|v|^{p} \phi \mathrm{d} x-\int_{\Omega} F_{v}(x, u, v) v \phi \mathrm{d} x\right]=\eta_{k}-\mu_{k} .
$$

Combing this with Lemma 3.1, we obtain $\left(\mu_{k}\right)^{p / p^{*}} S \leq \mu_{k}$. This result implies that

$$
\mu_{k}=0 \quad \text { or } \quad \mu_{k} \geq S^{p^{*} /\left(p^{*}-p\right)} .
$$

If the second case $\mu_{k} \geq S^{p^{*} /\left(p^{*}-p\right)}$ holds, for some $k \in J$, then by using Lemma 3.1 and the Hölder inequality, we have that

$$
\begin{aligned}
c & =\lim _{n \rightarrow \infty}\left(J_{n_{k}}\left(0, v_{n_{k}}\right)-\frac{1}{p^{*}}\left\langle\mathrm{~d} J_{n_{k}}\left(u_{n_{k}}, v_{n_{k}}\right),\left(0, v_{n_{k}}\right)\right\rangle\right) \\
& =\left(\frac{1}{p}-\frac{1}{p^{*}}\right) \int_{\Omega}\left(\left|\nabla v_{n_{k}}\right|^{p}+\left|v_{n_{k}}\right|^{p}\right) \mathrm{d} x-\int_{\Omega}\left[F\left(x, u_{n_{k}}, v_{n_{k}}\right)-\frac{1}{p^{*}} F_{v}\left(x, u_{n_{k}}, v_{n_{k}}\right) v_{n_{k}}\right] \mathrm{d} x \\
& \geq \frac{1}{N} \int_{\Omega} \mathrm{d} \mu-c\left(\frac{1}{2 N}\right)|\Omega| \\
& \geq \frac{1}{N} \int_{\Omega}\left|\nabla v_{n_{k}}\right|^{p} \mathrm{~d} x+\frac{1}{N} S^{p^{*} /\left(p^{*}-p\right)}-c\left(\frac{1}{2 N}\right)|\Omega| \\
& \geq \frac{1}{N} S^{p^{*} /\left(p^{*}-p\right)}-c\left(\frac{1}{2 N}\right)|\Omega|,
\end{aligned}
$$

where $\varepsilon=1 / 2 N$. This is impossible. Consequently, $\mu_{k}=0$ for all $k \in J$. From (3.8) we know that $\eta_{k}=0$ for all $k \in J$ and hence

$$
\int_{\partial \Omega}\left|v_{n_{k}}\right|^{p^{*}} \mathrm{~d} \sigma \rightarrow \int_{\partial \Omega}|v|^{p^{*}} \mathrm{~d} \sigma
$$

Now $v_{n_{k}} \rightarrow v$ in $E$ and Brezis-Lieb lemma [2] implies that

$$
\lim _{n \rightarrow \infty} \int_{\partial \Omega}\left|v_{n_{k}}-v\right|^{q^{*}} \mathrm{~d} \sigma=0
$$


Thus, we have

$$
\begin{aligned}
o(1)\left\|v_{n_{k}}\right\|_{p} & =\left\|v_{n_{k}}\right\|_{p}^{p}-\int_{\Omega}\left|v_{n_{k}}\right|^{p^{*}} \mathrm{~d} \sigma-\int_{\Omega} F_{v}\left(x, u_{n_{k}}, v_{n_{k}}\right) v_{n_{k}} \mathrm{~d} x \\
& =\left\|v_{n_{k}}-v\right\|_{p}^{p}+\|v\|_{p}^{p}-\int_{\Omega}|v|^{p^{*}} \mathrm{~d} \sigma-\int_{\Omega} F_{v}(x, u, v) v \mathrm{~d} x \\
& =\left\|v_{n_{k}}-v\right\|_{p}^{p}+o(1)\|v\|_{p},
\end{aligned}
$$

since $\mathrm{d} J_{n_{k}}(0, v)=0$. Thus we prove that $\left\{v_{n_{k}}\right\}$ strongly converges to $v$ in $E$. Thus (3.5) holds. (3.4) and (3.5) imply the conclusion of Lemma 3.4 follows.

\section{Proof of Theorem 1.1}

Proof of Theorem 1.1. Now we shall verify the conditions of Theorem 2.7. Obviously, $\left(B_{1}\right),\left(B_{2}\right),\left(B_{4}\right)$ in Theorem 2.7 are satisfied. Set $V_{j}=E_{j}=\operatorname{span}\left\{e_{1}, e_{2}, \ldots, e_{j}\right\}$, then $\left(B_{3}\right)$ is also satisfied. Since $1=\operatorname{dim} \widetilde{Y_{0}}<k_{0}=$ $\operatorname{dim} Y_{1},\left(B_{5}\right)$ is satisfied. In the following we verify the conditions in $\left(B_{7}\right)$. Since Fix $G \cap V=0$, that is $(a)$ of $\left(B_{7}\right)$ holds. It remains to verify $(b),(c)$ of $\left(B_{7}\right)$. Choose a number $\alpha$ such that

$$
\alpha<\min \left\{0, \frac{1}{N} S^{p^{*} /\left(p^{*}-p\right)}-c\left(\frac{1}{2 N}\right)|\Omega|, \frac{1}{N} 2^{\frac{p^{*}}{p-p^{*}}} S^{\frac{p p^{*}}{p-p^{*}}}-b\left(\frac{1}{2 p}\right)|\Omega|\right\} .
$$

(i) If $(0, v) \in Y_{0} \cap S_{\rho}$ (where $\rho$ is to be determined) then by $\left(H_{2}\right)$,

$$
\begin{aligned}
J(0, v) & =\frac{1}{p} \int_{\Omega}|\nabla v|^{p}+|v|^{p} \mathrm{~d} x-\frac{1}{p^{*}} \int_{\partial \Omega}|v|^{p^{*}} \mathrm{~d} \sigma-\int_{\Omega} F(x, 0, v) \mathrm{d} x \\
& \geq\left(\frac{1}{p}-\varepsilon\right) \cdot \int_{\Omega}|\nabla v|^{p}+|v|^{p} \mathrm{~d} x-\frac{1}{p^{*}} \int_{\partial \Omega}|v|^{p^{*}} \mathrm{~d} \sigma-b(\varepsilon)|\Omega| \\
& \geq \frac{1}{2 p}\|v\|_{p}^{p}-\frac{1}{p^{*}} S^{p^{*}}\|v\|_{p}^{p^{*}}-b\left(\frac{1}{2 p}\right)|\Omega|,
\end{aligned}
$$

where $\varepsilon=\frac{1}{2 p}$. Since

$$
\max _{t \in \mathbb{R}}\left(\frac{1}{2 p} t^{p}-\frac{1}{p^{*}} S^{p^{*}} t^{p^{*}}-b\left(\frac{1}{2 p}\right)|\Omega|\right)=\frac{1}{N} 2^{\frac{p^{*}}{p-p^{*}}} S^{\frac{p p^{*}}{p-p^{*}}}-b\left(\frac{1}{2 p}\right)|\Omega|,
$$

Therefore, there exists $\rho>0$ such that $J(0, v) \geq \alpha$ for every $\|v\|_{p}=\rho$, that is $(b)$ of $\left(B_{7}\right)$ holds.

(ii) For each $(u, v) \in U \oplus Y_{1}$, by condition $\left(H_{4}\right)$, we have

$$
\begin{aligned}
J(u, v)= & -\frac{1}{p} \int_{\Omega}\left(|\nabla u|^{p}+|u|^{p}\right) \mathrm{d} x+\frac{1}{p} \int_{\Omega}\left(|\nabla v|^{p}+|v|^{p}\right) \mathrm{d} x \\
& -\frac{1}{p^{*}} \int_{\partial \Omega}|u|^{p^{*}} \mathrm{~d} \sigma-\frac{1}{p^{*}} \int_{\partial \Omega}|v|^{p^{*}} \mathrm{~d} \sigma-\int_{\Omega} F(x, u, v) \mathrm{d} x \\
\leq & \frac{1}{p}\|v\|_{p}^{p}-L|v|_{p}^{p}+\xi|\Omega| \\
\leq & \max _{v \in E_{k_{0}}}\left(\frac{1}{p}\|v\|_{p}^{p}-L|v|_{p}^{p}\right)+\xi|\Omega| \\
= & \max _{\left\{t \geq 0, v \in \partial B_{1}(0) \cap E_{k_{0}}\right\}}\left[t^{p}\left(\frac{1}{p}-L|v|_{p}^{p}\right)\right]+\xi|\Omega|
\end{aligned}
$$


Let $r=\min \left\{\int_{\Omega}|v|^{p} \mathrm{~d} x: v \in \partial B_{1}(0) \cap E_{k_{0}}\right\}$. By taking $L \geq \frac{1}{p r}$, we have

$$
\frac{1}{p}-L|v|_{p}^{p} \leq \frac{1}{p}-L r \leq 0
$$

It follows from $(4.3),(4.4)$ and $\left(H_{4}\right)$ that

$$
J(u, v) \leq \xi|\Omega| \leq \min \left\{0, \frac{1}{N} S^{p^{*} /\left(p^{*}-p\right)}-c\left(\frac{1}{2 N}\right)|\Omega|\right\} .
$$

Let $\beta=\xi|\Omega|$, so we get $(c)$ in $\left(B_{7}\right)$. By Lemma 3.4, for any $c \in[\alpha, \beta], J(u, v)$ satisfies the condition of $(P S)_{c}^{*}$, then $\left(B_{6}\right)$ in Theorem 2.7 holds. So according to Theorem 2.7,

$$
c_{j}=\inf _{i \infty} \sup _{(A) \geq j} f(u), \quad-k_{0}+1 \leq j \leq-1,
$$

are critical values of $J, \alpha \leq c_{-k_{0}+1} \leq \cdots \leq c_{-1} \leq \beta<0$ and $J$ has at least $k_{0}-1$ pairs critical points.

Acknowledgements. The Project is supported by NSFC (10871096), Research Fundation during the 12st Five-Year Plan Period of Department of Education of Jilin Province, China (Grant [2011] No. 196), Natural Science Foundation of Changchun Normal University.

\section{REFERENCES}

[1] V. Benci, On critical point theory for indefinite functionals in presence of symmetries. Trans. Amer. Math. Soc. 274 (1982) $533-572$.

[2] H. Brézis and L. Nirenberg, Positive solutions of nonlinear elliptic equations involving critical exponents. Comm. Pure Appl. Math. 34 (1983) 437-477.

[3] M. Chipot, I. Shafrir and M. Fila, On the solutions to some elliptic equations with nonlinear boundary conditions. Advances Differential Equations 1 (1996) 91-110.

[4] J. Fernández Bonder and J.D. Rossi, Existence results for the p-Laplacian with nonlinear boundary conditions. J. Math. Anal. Appl. 263 (2001) 195-223.

[5] J. Fernández Bonder, J.P. Pinasco and J.D. Rossi, Existence results for a Hamiltonian elliptic system with nonlinear boundary conditions. Electron. J. Differential Equations 1999 (1999) 1-15.

[6] D.W. Huang and Y.Q. Li, Multiplicity of solutions for a noncooperative p-Laplacian elliptic system in $\mathbb{R}^{N}$. J. Differential Equations 215 (2005) 206-223.

[7] W. Krawcewicz and W. Marzantowicz, Some remarks on the Lusternik-Schnirelman method for non-differentiable functionals invariant with respect to a finite group action. Rocky Mt. J. Math. 20 (1990) 1041-1049.

[8] Y.Q. Li, A limit index theory and its application. Nonlinear Anal. 25 (1995) 1371-1389.

[9] F. Lin and Y.Q. Li, Multiplicity of solutions for a noncooperative elliptic system with critical Sobolev exponent. Z. Angew. Math. Phys. 60 (2009) 402-415.

[10] J. Lindenstrauss and L. Tzafriri, Classical Banach Spaces I. Springer, Berlin (1977).

[11] P.L. Lions, The concentration-compactness principle in the caculus of variation: the limit case, I. Rev. Mat. Ibero. 1 (1985) 45-120.

[12] P.L. Lions, The concentration-compactness principle in the caculus of variation: the limit case, II. Rev. Mat. Ibero. 1 (1985) $145-201$.

[13] K. Pflüger, Existence and multiplicity of solutions to a $p$-Laplacian equation with nonlinear boundary condition, Electron. J. Differential Equations 10 (1998) 1-13.

[14] S. Terraccini, Symmetry properties of positive solutions to some elliptic equations with nonlinear boundary conditions. Differential Integral Equations 8 (1995) 1911-1922.

[15] H. Triebel, Interpolation Theory, Function Spaces, Differential Operators. North- Holland, Amsterdam (1978).

[16] M. Willem, Minimax Theorems. Birkhäuser, Boston (1996). 